This item was submitted to Loughborough's Research Repository by the author.

Items in Figshare are protected by copyright, with all rights reserved, unless otherwise indicated.

\title{
Ischemic preconditioning enhances critical power during a 3 minute all-out cycling test
}

PLEASE CITE THE PUBLISHED VERSION

http://dx.doi.org/10.1080/02640414.2017.1349923

PUBLISHER

(C) Taylor and Francis

\section{VERSION}

AM (Accepted Manuscript)

\section{PUBLISHER STATEMENT}

This work is made available according to the conditions of the Creative Commons Attribution-NonCommercialNoDerivatives 4.0 International (CC BY-NC-ND 4.0) licence. Full details of this licence are available at: https://creativecommons.org/licenses/by-nc-nd/4.0/

\section{LICENCE}

CC BY-NC-ND 4.0

\section{REPOSITORY RECORD}

Griffin, Patrick J., Richard A. Ferguson, Conor Gissane, Stephen J. Bailey, and Stephen D. Patterson. 2019. "Ischemic Preconditioning Enhances Critical Power During a 3 Minute All-out Cycling Test". figshare. https://hdl.handle.net/2134/25837. 
Ischemic preconditioning enhances critical power during a 3 minute all-out cycling test

Patrick J. Griffin ${ }^{1}$, Richard A. Ferguson ${ }^{2}$, Conor Gissane ${ }^{1}$, Stephen J. Bailey ${ }^{2}$, Stephen D. Patterson ${ }^{1}$

1. School of Sport, Health, and Applied Science, St. Mary's University, London, UK

2. School of Sport, Exercise and Health Sciences, Loughborough University, Loughborough, UK

Corresponding Author:

Dr Stephen D. Patterson

School of Sport, Health and Applied Sciences, St Mary's University, Waldegrave Road, Strawberry Hill, Twickenham, UK

Phone: +44 2082402357

Fax: +442082404212

E-mail: Stephen.Patterson@stmarys.ac.uk

Running Title: Ischemic preconditioning enhances critical power

Abstract word count: 200

Word Count: 3834 


\section{ABSTRACT}

This study tested the hypothesis that ischemic preconditioning (IPC) would increase critical power (CP) during a 3 minute all-out cycling test. Twelve males completed two 3 minute allout cycling tests, in a crossover design, separated by 7 days. These tests were preceded by IPC (4 x 5 minute intervals at $220 \mathrm{mmHg}$ bilateral leg occlusion) or SHAM treatment (4 x 5 minute intervals at $20 \mathrm{mmHg}$ bilateral leg occlusion). CP was calculated as the mean power output during the final $30 \mathrm{~s}$ of the 3 minute test with $\mathrm{W}^{\prime}$ taken as the total work done above CP. Muscle oxygenation was measured throughout the exercise period. There was a $15.3 \pm$ 0.3\% decrease in muscle oxygenation (TSI; [Tissue saturation index]) during the IPC stimulus, relative to SHAM. CP was significantly increased ( $241 \pm 65 \mathrm{~W}$ vs. $234 \pm 67 \mathrm{~W})$, whereas $\mathrm{W}^{\prime}(18.4 \pm 3.8 \mathrm{vs} 17.9 \pm 3.7 \mathrm{~kJ})$ and total work done (TWD) were not different $(61.1$ \pm 12.7 vs $60.8 \pm 12.7 \mathrm{~kJ}$ ), between the IPC and SHAM trials. IPC enhanced CP during a 3 minute all-out cycling test without impacting $\mathrm{W}^{\prime}$ or TWD. The improved CP after IPC might contribute towards the effect of IPC on endurance performance.

Key Words: W Prime; High intensity exercise; near-infrared spectroscopy; priming; occlusion. 


\section{INTRODUCTION}

Ischemic preconditioning (IPC) is a technique that applies a blood pressure cuff or tourniquet to a limb to induce repeated cycles of brief ischemia followed by reperfusion (de Groot et al. 2010). IPC has been shown to improve exercise performance across a range of exercise tasks (de Groot et al. 2010, Barbosa et al. 2015, Crisafulli et al. 2011, Kjeld et al. 2014, Jean-StMichel et al. 2011, Patterson et al. 2015), but existing evidence suggests that IPC is more likely to improve performance during endurance exercise tests that rely predominantly on aerobic metabolism (de Groot et al. 2010, Barbosa et al. 2015, Crisafulli et al. 2011, Kjeld et al. 2014, Bailey et al. 2012b, Kido et al. 2015), compared to short-duration high-intensity exercise tests that mandate a high anaerobic energy turnover (Crisafulli et al. 2011, Gibson et al. 2015, Paixao, da Mota \& Marocolo 2014). However, while IPC is more likely to be ergogenic during endurance exercise, the mechanisms that underlie the ergogenic effects of IPC are unclear.

An important determinant of endurance exercise performance and the maximal sustainable rate of aerobic metabolism is the critical power (CP; Poole et al. 2016). Indeed CP, the asymptote of the hyperbolic power-duration relationship, has been reported to represent the highest power output that can be sustained without a progressive loss of homeostasis (Poole et al. 2016, Moritani et al. 1981). On the other hand, the curvature constant of the powerduration hyperbola, $\mathrm{W}^{\prime}$, is a parameter predominantly underpinned by anaerobic metabolism (Moritani et al. 1981). Importantly, the tolerable duration of high-intensity exercise, or the time required to complete a set amount of work, can be accurately predicted from the interaction between CP and $\mathrm{W}^{\prime}$ (Vanhatalo, Doust \& Burnley 2007). Accordingly, the improved endurance performance previously reported after IPC might be linked to an increase in CP and/or $\mathrm{W}^{\prime}$. 
Studies using near-infrared spectroscopy (NIRS) have reported that skeletal muscle [deoxyhaemoglobin] ([HHb]) is increased after IPC (Barbosa et al. 2015, Kjeld et al. 2014, Kido et al. 2015, Bailey et al. 2012a), suggestive of an increase in muscle $\mathrm{O}_{2}$ extraction (Grassi et al. 2003). Interventions that increase muscle $\mathrm{O}_{2}$ extraction have the potential to speed $\dot{V} \mathrm{O}_{2}$ kinetics (Breese et al. 2013), which has been associated with an increase in CP (Vanhatalo et al. 2010, Murgatroyd et al. 2011). In addition, IPC has been suggested to increase production of nitric oxide (NO; Rassaf et al. 2014), a potent vasoactive metabolite. Therefore, IPC might also have the potential to increase skeletal muscle $\mathrm{O}_{2}$ delivery during exercise. The CP has been shown to be sensitive to $\mathrm{O}_{2}$ delivery as evidenced by increased CP when $\mathrm{O}_{2}$ delivery is elevated (Vanhatalo et al. 2010) and lower $\mathrm{CP}$ when $\mathrm{O}_{2}$ delivery is attenuated (Broxterman et al. 2015). Therefore, IPC has the potential to increase skeletal muscle $\mathrm{O}_{2}$ supply and utilisation. These positive changes in skeletal muscle $\mathrm{O}_{2}$ supply and utilisation might positively impact on $\mathrm{CP}$ promoting enhanced endurance exercise performance. However, this has yet to be investigated. The aim of the present study was to investigate the effect of IPC on CP and $\mathrm{W}^{\prime}$ assessed using a 3 minute all-out cycling test. NIRS was used to provide information on the balance between skeletal muscle $\mathrm{O}_{2}$ supply and utilisation, to explore the potential mechanisms for any improvements in $\mathrm{CP}$ and $\mathrm{W}^{\prime}$ following IPC. It was hypothesised that IPC would increase CP in association with a higher TSI, without altering $\mathrm{W}^{\prime}$. 


\section{METHODS}

Twelve recreationally-active males (age $30 \pm 6$ years; stature $1.8 \pm 0.1 \mathrm{~m}$; body mass $82.1 \pm$ $10.7 \mathrm{~kg}$; sum of 7-sites $74.1 \pm 19.3 \mathrm{~mm} ; \dot{\mathrm{VO}}_{2}$ peak $4.18 \pm 0.86 \mathrm{~L} \cdot \mathrm{min}-1$; Training time per week $7 \pm 3 \mathrm{hr}$; mean \pm SD) volunteered to participate in the experiment. Participants were informed of the nature of the research, but remained naïve to the study rationale to remove any placebo effect of IPC, and provided written informed consent to participate in the study. Participants were clear of contraindications that would preclude them from participating in high intensity exercise after the application of an IPC protocol. Approval for the study was granted by the St. Mary’s University ethics committee, which conformed to the Declaration of Helsinki.

All participants reported to the laboratory on five separate occasions to perform an incremental ramp cycle ergometer test for the determination of $\dot{\mathrm{V}}_{2}$ peak and gas exchange threshold (GET), two 3 minute all-out test familiarisation trials, and in a single blind, counterbalanced, crossover study design two trials consisting of IPC or SHAM interventions prior to a 3 minute all-out test. A minimum of $48 \mathrm{~h}$ rest separated each of the initial incremental and familiarisation tests, and a minimum of seven days separated the intervention trials to eliminate possible carryover of acute IPC (Loukogeorgakis et al. 2005). All testing was completed within 21 days in the same laboratory (temperature: $18 \pm 1^{\circ} \mathrm{C}$ ) at the same time of day $\pm 1 \mathrm{~h}$. Throughout the experimental period, participants were instructed to follow their normal diet and fluid intake habits, with a specific request to refrain from caffeine, alcohol, and exercise, $24 \mathrm{~h}$ prior to testing.

During the first visit, anthropometric measurements of participants were obtained and the cycle ergometer was individually set up, with measurements recorded and replicated for all subsequent trials. Participants then performed an incremental ramp cycling protocol on an 
electronically-braked cycle ergometer (Lode Excalibur Sport, Lode BV, Groningen, Netherlands) to assess $\mathrm{VO}_{2}$ peak and GET. The protocol consisted of a three minute unloaded baseline pedalling warm up phase, followed immediately by a $30 \mathrm{~W} \cdot \mathrm{min}^{-1}$ ramp protocol. Participants self-selected a cadence between 75 - 95 rpm during the unloaded period, where they cycled freely for the initial $60 \mathrm{~s}$, confirming their comfort at that cadence. The test ended at volitional exhaustion or was terminated when the self-selected pedal cadence reduced by 5 rpm for more than 10 seconds. Pulmonary gas exchange was measured breath-by-breath throughout the test. $\dot{\mathrm{V}} \mathrm{O}_{2}$ peak was taken as the highest $30 \mathrm{~s}$ average recorded during the test, with $\dot{\mathrm{VO}}_{2}$ and $\dot{\mathrm{V} C O}$ data reduced to 5 s intervals for the estimation of the GET using the Vslope method (Beaver, Wasserman \& Whipp 1986; Bailey et al. 2009). Briefly, two researchers performed independent analysis of GET using the following cluster of measurements; a) the first disproportionate increase in $\mathrm{CO}_{2}$ production from visual inspection of individual plots of $\dot{V} \mathrm{CO}_{2}$ vs. $\dot{V} \mathrm{O}_{2} ;$ b) an increase in expired ventilation ( $\left.\dot{V} \mathrm{E}\right) / \dot{V} \mathrm{O}_{2}$ with no increase in $\dot{V} \mathrm{E} / \dot{V} \mathrm{CO}_{2}$; and 3) an increase in end-tidal $\mathrm{O}_{2}$ tension with no fall in end-tidal $\mathrm{CO}_{2}$ tension. To account for the time delay in the increase in $\dot{V} \mathrm{O}_{2}$ to the increase in the external work-rate during incremental exercise, the work-rate corresponding to GET was reduced by two thirds of the ramp rate (i.e. $20 \mathrm{~W}$; Whipp, 1987).

During the next two visits, participants performed the 3 minute all-out exercise test as described below. For the main experimental trials, participants arrived at the laboratory for initial assessment of body mass. Thereafter, participants were required to lie in a semi-supine position on an adjustable medical couch where resting blood pressure was measured using an automated sphygmomanometer (Nissei DS-500, Japan Precision Instruments Inc., Gunma, Japan). During this period a wireless portable NIRS device (PortaMon MK II, Artinis Medical Systems, The Netherlands) was placed and prepared as described below. This allowed for the measurement of muscle oxygenation variables (HHb and TSI). Pneumatic 
pressure cuffs (14.5 cm width - Delfi Medical Innovations, Vancouver, Canada) were then applied bilaterally and simultaneously to the proximal portion of the upper leg and inflated for 5 minutes followed by 5 minutes of reperfusion. This was repeated four times, lasting a total of 40 minutes. In the IPC condition the cuffs were inflated to $220 \mathrm{mmHg}$ (Kooijman et al. 2008) to ensure circulatory occlusion of the limb whilst for the SHAM condition the cuffs were inflated to $20 \mathrm{mmHg}$ (Bailey et al. 2012b).

The test protocol began immediately after the IPC/SHAM treatment, with a 3 minute resting period with the participant sitting quietly on the ergometer and their right leg hanging in a relaxed manner. Following this, they immediately began a 5 minute warm up at 90\% GET (performed at the participant's previously self-selected cadence), followed by 5 minutes of seated passive recovery. Participants then completed a pre-test, 3 minute of unloaded cycling at their previously self-selected cadence with the final $10 \mathrm{~s}$ of this period used to increase cadence to $110 \mathrm{rpm}$ providing a 'rolling start' for the following all-out effort. A count down was provided to coincide with the start of the 3 minute all-out cycling test. At the beginning of this test participants were asked to provide a maximal effort and then sustain as high a cadence as possible. Strong verbal encouragement was provided throughout however, elapsed time feedback, displays of power output and cadence were hidden to prevent the participants from employing pacing strategies. Following the end of the test participants were advised to remain seated on the bike and cycle at a self-selected power output for 10 minutes.

$\mathrm{CP}$ was calculated as the average power output in the final $30 \mathrm{~s}$ of the 3 minute test. $\mathrm{W}^{\prime}$ was calculated as the power-time integral above CP across the 3 minutes of work. TWD was calculated as the sum of 3 minutes of work done (Burnley, Doust \& Vanhatalo 2006), whilst the peak power output achieved during the test was defined as the highest power output over a 5 s period during the test. To assess the potential impact of IPC on performance, estimates of $\mathrm{CP}$ and $\mathrm{W}^{\prime}$ were used to predict the time taken to complete a range of work targets $(50,75$, 
$100,125,150,175,200,225,250 \mathrm{KJ})$, as previously described and using the following equation (Kelly et al. 2013):

$\operatorname{Tlim}=\left(\mathrm{W}-\mathrm{W}^{\prime}\right) / \mathrm{CP}$.

Pulmonary gas exchange was measured throughout the entire protocol using a breath-bybreath open spirometric system (Oxycon Pro, Jaeger Ltd., Hoechberg, Germany). The turbine volume transducer was calibrated using a $3 \mathrm{~L}$ precision syringe (Hans Rudolph Inc., Shawnee, USA), and the gas analyser calibrated prior to each trial using $\mathrm{O}_{2}$ and $\mathrm{CO}_{2}$ of known concentration (Cryoserve, Worcester, UK). Prior to analysis the breath-by-breath $\dot{V} \mathrm{O}_{2}$ data from each test were treated as described previously (Bailey et al. 2011). A singleexponential model without time delay, with the fitting window commencing at $t=0 \mathrm{~s}$ (equivalent to the mean response time, MRT) was used to characterize the kinetics of the overall $\dot{V} \mathrm{O}_{2}$ response during the trials, using an iterative process, as described in the following equation:

$\dot{V} \mathrm{O}_{2}(\mathrm{t})=\dot{V} \mathrm{O}_{2}$ baseline $+\mathrm{A}\left(1-\mathrm{e}^{-(\mathrm{t} / \mathrm{MRT})}\right)$

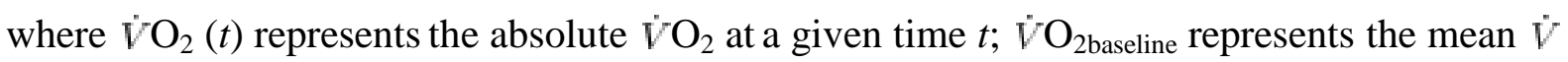
$\mathrm{O}_{2}$ measured over the final 60-s of baseline; and A and MRT represent the amplitude and MRT, respectively, describing the overall increase in $\dot{V} \mathrm{O}_{2}$ above baseline. The absolute $\dot{V} \mathrm{O}_{2}$ at end-exercise (average over the final 30-s) and the total $\mathrm{O}_{2}$ consumed were also calculated. Blood [lactate] was analysed at rest and $60 \mathrm{~s}$ post-exercise via earlobe capillary blood samples, collected using a heparinized $20 \mu$ end-to-end capillary tube and assessed in an automatic analyser (Biosen C-Line lactate analyser, EKF Diagnostics, Penarth, UK; CV = 1.5\%). It should be noted that the blood [lactate] value $60 \mathrm{~s}$ post-exercise would not necessarily reflect the peak value from the test. Heart rate (HR) was measured continuously 
via telemetry (RS610, Polar Electro Japan) with a whole period average value calculated for the cycling exercise periods.

A wireless portable NIRS unit was located on the vastus lateralis muscle belly of the right leg. The location was identified with the participant in a semi-recumbent sitting pose, an 11 cm dowel was placed under their right knee, and a mark placed 2/3 of the distal distance between the anterior superior iliac spine and tibiale laterale (Patterson et al. 2015). The unit was set to record at $10 \mathrm{~Hz}$, with data converted to $1 \mathrm{~s}$ intervals and averaged over the final 30

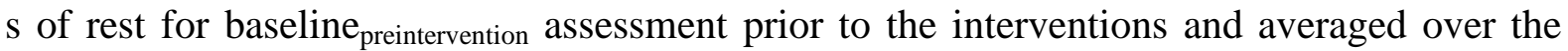
accumulated 20 minutes of IPC/SHAM occlusion and reperfusion periods. The [HHb] kinetics during the exercise bouts was determined by fitting a mono-exponential model with a time delay (TD) from the nadir of the initial decline in the [HHb] response to the point at which mono-exponentially became distorted, consequent to a gradual fall in [HHb], as determined by visual inspection of the residual plots. The TSI values at baseline postintervention were averaged over 60-s preceding the onset of the trial, as well as the first 60 -s and 120 -s and last 30 -s of the 180 -s trial.

Data were analysed using the Statistical Package for the Social Sciences (SPSS v21). All data are presented as mean \pm SD. Shapiro-Wilk and Kolmogorov-Smirnov tests indicated a normal distribution of data. The differences between IPC and sham for CP, $\mathrm{W}^{\prime}$ and physiological measures were assessed via a two tailed paired Student's $t$-test. The predicted performance times in IPC and SHAM conditions were analysed using a two-way repeated measures ANOVA (2 conditions x 8 workloads) with significant main effects further analysed using Tukeys honest significant difference (HSD). The level of significance was set at $\mathrm{p}<0.05$ for all analyses. 


\section{RESULTS}

$\dot{\mathrm{V}} \mathrm{O}_{2 \text { peak }}$ and power output at $\dot{\mathrm{VO}}_{2 \text { peak }}$ were $4.06 \pm 0.79 \mathrm{~L} \cdot \mathrm{min}^{-1}$ and $344 \pm 52 \mathrm{~W}$ respectively, with GET occurring at $2.33 \pm 0.38 \mathrm{~L} \cdot \mathrm{min}^{-1}$ (equivalent to $59 \pm 10 \%$ of $\dot{\mathrm{VO}}_{2 \text { peak }}$ ) and $162 \pm 38$ W. $\dot{\mathrm{V}} \mathrm{O}_{2 \text { peak }}$ during the 3 minute all-out test was $97 \%$ and $95 \%$ of the $\dot{\mathrm{V}} \mathrm{O}_{2 \text { peak }}$ value recorded in the ramp test for the IPC and SHAM trials. Furthermore $\mathrm{HR}_{\max }$ during the 3 minute all-out test represented $98 \%$ and $97 \%$ of the values achieved in the ramp test for IPC and SHAM, respectively.

$\mathrm{CP}$ was $7 \pm 2 \mathrm{~W}$ greater following IPC compared to SHAM (Table 1, Figure 1). The coefficient of variation (CV) of the CP in 3 minute all-out test was 2.4\% (5.5 W). There were no differences in $\mathrm{W}^{\prime}$ or TWD between IPC and SHAM (Table 1). Nor were there any differences in $\dot{\mathrm{V}} \mathrm{O}_{2 \text { peak }}$, total $\mathrm{O}_{2}$ consumed, $\dot{\mathrm{V}} \mathrm{O}_{2} \mathrm{MRT}$, peak power output, post-exercise blood [lactate], $\mathrm{HR}_{\max }$ or $\mathrm{HR}_{\text {mean }}$ between IPC and SHAM (Table 1).

When $\mathrm{CP}$ and $\mathrm{W}^{\prime}$ were combined to predict performance across a number of work done targets, there were significant main effects for condition and work done and a significant interaction $(\mathrm{P}<0.05)$. Post-hoc analysis revealed a significant improvement in performance (2.9\% - 3.3\%) for workloads 100-250kJ but not below 75 kJ (Figure 2).

There were no differences in baseline TSI between IPC and SHAM (Table 2). The IPC intervention resulted in significantly lower TSI values during the 20 minute occlusion period compared to SHAM (Table 2). There were no differences in muscle TSI during the 3 minute all-out test between IPC and SHAM, and no significant difference for $\mathrm{HHb}$ TD $+\tau$ during exercise $(4.3 \pm 3.0$ vs $6.6 \pm 4.1, \mathrm{p}=0.081)$. 


\section{DISCUSSION}

The main findings of this study were that a bilateral IPC stimulus of the lower limbs increased CP by $7 \mathrm{~W}$ ( 3\%), but had no effect on $\mathrm{W}^{\prime}$ or TWD, during a 3 minute all-out test compared to a SHAM condition. These observations are consistent with our hypotheses. The increased CP was predicted to improve endurance exercise performance, particularly during longer duration trials, as demonstrated in figure 2. These findings suggest that IPC can improve $\mathrm{CP}$ without impacting $\mathrm{W}^{\prime}$, and is more likely to enhance performance in events where the mean power output is in the lower compared to the upper limits of the severeintensity exercise domain.

The predicted 2-3\% improvement in endurance performance in this study supports a number of studies demonstrating enhanced performance after IPC, including a $2.5 \%$ improvement in $5 \mathrm{~km}$ time trial performance (Bailey et al. 2012b) and 1\% improvement in $1 \mathrm{~km}$ rowing ergometer time trial performance (Kjeld et al. 2014). In the current study, CP was increased concomitant with no increase in $\dot{\mathrm{V}} \mathrm{O}_{2 \text { peak }}$ or $\mathrm{W}^{\prime}$ between the IPC and SHAM trials. Although the improvement in CP was small (3\%), the potential practical significance of this change can be recognised if one considers that the inter-relationship between $\mathrm{CP}$ and $\mathrm{W}^{\prime}$ can be used to accurately predict performance times within the severe intensity domain (Kelly et al. 2013). Indeed, the 3\% increase in CP with IPC in this study was predicted to translate into a 2-3\% improvement in the time to complete a fixed amount of work, except for the two lowest work targets. Therefore, these findings suggest that IPC has the potential to improve time trial performance in the severe intensity domain, consequent to enhanced CP. Consistent with this postulate, CP derived from the 3 minute all-out test has been shown to predict $16.1 \mathrm{~km}$ road time trial performance (Black et al. 2014), however it is unlikely that it could be used to directly predict performance time as this was $>25$ min so likely not in the severe-intensity domain for some athletes. Increasing CP therefore would allow an individual to cycle, in the 
severe intensity domain, at a higher power output before a precipitous increase in fatigue related metabolites and decline in finite anaerobic energy reserves occurs (Vanhatalo et al. 2016, Poole et al. 1988, Jones et al. 2008), which might underlie any improvements in exercise performance after IPC.

In line with our experimental hypothesis, the IPC protocol, which provided 20 minutes of occlusion, resulted in a 15.3\% reduction in TSI (a marker of the dynamic balance between muscle $\mathrm{O}_{2}$ supply and consumption) compared with the SHAM intervention. IPC has been shown to increase circulating concentrations of nitrite, a key physiological signalling molecule and marker of endothelial nitric oxide synthase (eNOS) activity, through the sheer stress imposed on the vascular endothelium during the reperfusion period and the resultant activation of eNOS (Rassaf et al. 2014). Nitrite, and its potential to undergo $\mathrm{O}_{2}$-independent reduction to NO, has several biological functions including the regulation of vascular tone, blood flow and mitochondrial respiration (Stamler, Meissner 2001). Therefore, IPC has the potential to influence muscle oxygenation responses, as evidenced by greater muscle oxygenation in rats during exercise (Saito et al. 2004) and faster HHb kinetics during moderate-intensity cycling exercise (Kido et al. 2015). Moreover, greater deoxygenated haemoglobin and myoglobin concentrations have been observed following IPC in human skeletal muscle during handgrip exercise, suggesting increased $\mathrm{O}_{2}$ extraction (Barbosa et al. 2015). However, there were no differences in TSI or HHb kinetics between conditions during the 3 minute all-out test in the present study.

IPC had no effect on $\mathrm{W}^{\prime}$, and since $\mathrm{W}^{\prime}$ is closely associated with the depletion of finite anaerobic energy reserves and the accumulation of metabolites derived from anaerobic energy pathways (Miura, Sato, Whipp, \& Fukuba, 2000), our finding suggests that IPC did not measurably impact anaerobic energy processes during the $3 \mathrm{~min}$ all out test. This observation supports previous research which has demonstrated no improvement in 
performance following IPC when anaerobic metabolism was the predominant energy system utilised (Gibson et al. 2015; Paixão et al., 2014). In contrast, Patterson and colleagues (2015) demonstrated peak and mean power output were increased in the early stages of a land based repeated sprint protocol, which demonstrated improved performance in the early stages following IPC. It should be noted that there are some important differences between this current study and previous work, particularly with regard to the timing of the IPC intervention. In the current study the exercise commenced within 8 minutes of the last occlusion cycle which is a similar time frame to previous work showing no effect of IPC on anaerobic performance (Gibson et al 2015; Paixão et al., 2014). In contrast, work from Patterson et al. (2015) demonstrated improved anaerobic performance 45 minutes post IPC application and a recent meta-analysis suggests this time frame has a larger effect on anaerobic performance (Salvador et al. 2016). Taken together, these findings suggest that the timing of the IPC intervention may have implications for the metabolic pathways and component of the hyperbolic power-duration relationship that are potentiated following IPC administration.

IPC enhances CP which may translate to improved exercise performance particularly in the severe intensity domain. The \% change for CP was greater than the CV (2.4\%), indicating that the effect was greater than the typical measurement error. Alongside the improvement in $\mathrm{CP}$, the changes in predicted performance across a range of time trials (2.9 - 3.3\%) may be practically meaningful as they are larger than the $0.6 \%$ value suggested to be the smallest 'worthwhile' improvement for road time trial cyclists (Paton, Hopkins 2001). Therefore, our data support the use of IPC as a pre competition intervention to enhance endurance exercise performance.

There are several limitations with this study. When fitting pulmonary $\dot{\mathrm{VO}}_{2}$ kinetics, researchers normally average several bouts of exercise in order to reduce the signal to noise 
ratio. In the current study we only employed a single bout of exercise. In this case, we used a mean response time to give an appreciation of the overall response dynamics, rather than attempting to precisely characterise the phase II time constant. Since the amplitude is large the confidence in the fitting procedure is enhanced as there are more data points to fit the exponential through. Furthermore we also used one exercise bout to fit muscle NIRS kinetics. Kido et al. (2015) suggest that one trial may be sufficient to model muscle kinetics. Due to the high frequency of sampling $(10 \mathrm{~Hz})$ we believe that modelling NIRS kinetics are reliable

even with one trial. Furthermore, one transition is appropriate as the data is less noisy, compared to breath-by-breath $\dot{\mathrm{VO}}_{2}$ data so the signal to noise ratio is improved. A further limitation of NIRS is that increased skin perfusion might influence light absorbance and scattering and, thus, the NIRS-derived variables. However, Tew et al. (2010) demonstrated that while thigh heating increased cutaneous vascular conductance and vastus lateralis total haemoglobin concentration, muscle oxygenation was increased with heating at rest but not during exercise. On the other hand, Messere and Roatta (2013) observed no change in muscle oxygenation with local heating at rest or during exercise. Therefore, the effects of skin blood flow on TSI are controversial at rest, but less so during exercise. Future research should focus on investigating the pulmonary and muscle kinetics with multiple exercise bouts following IPC. Furthermore emphasis should be placed on optimising the most appropriate time that IPC can be applied before competition / performance. Alongside this more focus should be placed on the plasma and muscle mediators that may underlie the physiological and performance effects of IPC.

\section{CONCLUSIONS}

In conclusion, our findings suggest that IPC can improve $\mathrm{CP}$, but not $\mathrm{W}^{\prime}$, during a 3 minute all-out test in healthy men. The increase in CP in the IPC trial was predicted to evoke practically meaningful improvements in cycling time trial performance when the work to be 
completed is in the range of $100-250 \mathrm{~kJ}$ (Figure 2). Therefore, our study supports the use of IPC as a novel, cost-effective intervention to improve CP with potential to enhance endurance exercise performance in humans. 


\section{Acknowledgements}

The authors would like to thank all the participants for their enthusiasm and dedication to the project. No financial assistance has been provided for this study. 


\section{REFERENCES}

Bailey, S. J., Wilkerson, D. P., DiMenna, F. J., \& Jones, A. M. (2009). Influence of repeated sprint training on pulmonary $\mathrm{O} 2$ uptake and muscle deoxygenation kinetics in humans. Journal of Applied Physiology, 106, 1875-1887

Bailey, S.J., Vanhatalo, A., DiMenna, F.J., Wilkerson, D.P. \& Jones, A.M. (2011). Fast-start strategy improves $\mathrm{VO}_{2}$ kinetics and high-intensity exercise performance. Medicine and science in sports and exercise, 43(3), 457-467.

Bailey, T.G., Birk, G.K., Cable, N.T., Atkinson, G., Green, D.J., Jones, H. \& Thijssen, D.H. (2012a). Remote ischemic preconditioning prevents reduction in brachial artery flowmediated dilation after strenuous exercise. American journal of physiology.Heart and circulatory physiology, 303(5), H533-8.

Bailey, T.G., Jones, H., Gregson, W., Atkinson, G., Cable, N.T. \& Thijssen, D.H. (2012b). Effect of ischemic preconditioning on lactate accumulation and running performance. Medicine and science in sports and exercise, 44(11), 2084-2089.

Barbosa, T.C., Machado, A.C., Braz, I.D., Fernandes, I.A., Vianna, L.C., Nobrega, A.C. \& Silva, B.M. (2015). Remote ischemic preconditioning delays fatigue development during handgrip exercise. Scandinavian Journal of Medicine \& Science in Sports, 25(3), 356-364.

Beaver, W.L., Wasserman, K. \& Whipp, B.J. (1986). A new method for detecting anaerobic threshold by gas exchange. Journal of applied physiology (Bethesda, Md.: 1985), 60(6), 2020-2027.

Black, M.I., Durant, J., Jones, A.M. \& Vanhatalo, A. (2014). Critical power derived from a 3min all-out test predicts 16.1-km road time-trial performance. European journal of sport science, 14(3), 217-223.

Breese, B.C., McNarry, M.A., Marwood, S., Blackwell, J.R., Bailey, S.J. \& Jones, A.M. (2013). Beetroot juice supplementation speeds $\mathrm{O}_{2}$ uptake kinetics and improves exercise tolerance during severe-intensity exercise initiated from an elevated metabolic rate. American journal of physiology.Regulatory, integrative and comparative physiology, 305(12), R1441-50.

Broxterman, R.M., Craig, J.C., Smith, J.R., Wilcox, S.L., Jia, C., Warren, S. \& Barstow, T.J. (2015). Influence of blood flow occlusion on the development of peripheral and central fatigue during small muscle mass handgrip exercise. The Journal of physiology, 593(17), 4043-4054.

Burnley, M., Doust, J.H. \& Vanhatalo, A. (2006). A 3-min all-out test to determine peak oxygen uptake and the maximal steady state. Medicine and science in sports and exercise, 38(11), 1995-2003.

Crisafulli, A., Tangianu, F., Tocco, F., Concu, A., Mameli, O., Mulliri, G. \& Caria, M.A. (2011). Ischemic preconditioning of the muscle improves maximal exercise performance 
but not maximal oxygen uptake in humans. Journal of applied physiology (Bethesda, Md.: 1985), 111(2), 530-536.

de Groot, P.C., Thijssen, D.H., Sanchez, M., Ellenkamp, R. \& Hopman, M.T. (2010). Ischemic preconditioning improves maximal performance in humans. European journal of applied physiology, 108(1), 141-146.

Gibson, N., Mahony, B., Tracey, C., Fawkner, S. \& Murray, A. (2015). Effect of ischemic preconditioning on repeated sprint ability in team sport athletes. Journal of sports sciences, 33(11), 1182-1188.

Grassi, B., Pogliaghi, S., Rampichini, S., Quaresima, V., Ferrari, M., Marconi, C. \& Cerretelli, P. (2003). Muscle oxygenation and pulmonary gas exchange kinetics during cycling exercise on-transitions in humans. Journal of applied physiology (Bethesda, Md.: 1985), 95(1), 149-158.

Jean-St-Michel, E., Manlhiot, C., Li, J., Tropak, M., Michelsen, M.M., Schmidt, M.R., McCrindle, B.W., Wells, G.D. \& Redington, A.N. (2011). Remote preconditioning improves maximal performance in highly trained athletes. Medicine and science in sports and exercise, 43(7), 1280-1286.

Jones, A.M., Wilkerson, D.P., DiMenna, F., Fulford, J. \& Poole, D.C. (2008). Muscle metabolic responses to exercise above and below the "critical power" assessed using 31PMRS. American journal of physiology.Regulatory, integrative and comparative physiology, 294(2), R585-93.

Kelly, J., Vanhatalo, A., Wilkerson, D.P., Wylie, L.J. \& Jones, A.M. (2013). Effects of nitrate on the power-duration relationship for severe-intensity exercise. Medicine and science in sports and exercise, 45(9), 1798-1806.

Kido, K., Suga, T., Tanaka, D., Honjo, T., Homma, T., Fujita, S., Hamaoka, T. \& Isaka, T. (2015). Ischemic preconditioning accelerates muscle deoxygenation dynamics and enhances exercise endurance during the work-to-work test. Physiological reports, 3(5), 10.14814/phy2.12395.

Kjeld, T., Rasmussen, M.R., Jattu, T., Nielsen, H.B. \& Secher, N.H. (2014). Ischemic preconditioning of one forearm enhances static and dynamic apnea. Medicine and science in sports and exercise, 46(1), 151-155.

Kooijman, M., Thijssen, D.H., de Groot, P.C., Bleeker, M.W., van Kuppevelt, H.J., Green, D.J., Rongen, G.A., Smits, P. \& Hopman, M.T. (2008). Flow-mediated dilatation in the superficial femoral artery is nitric oxide mediated in humans. The Journal of physiology, 586(4), 1137-1145.

Loukogeorgakis, S.P., Panagiotidou, A.T., Broadhead, M.W., Donald, A., Deanfield, J.E. \& MacAllister, R.J. (2005). Remote ischemic preconditioning provides early and late protection against endothelial ischemia-reperfusion injury in humans: role of the 
autonomic nervous system. Journal of the American College of Cardiology, 46(3), 450456.

Messere, A. \& Roatta, S. (2013). Influence of cutaneous and muscular circulation on spatially resolved versus standard Beer-Lambert near-infrared spectroscopy. Physiological Reports, 1(7), e00179.

Miura, A., Sato, H., Whipp, B. J., \& Fukuba, Y. (2000). The effect of glycogen depletion on the curvature constant parameter of the power-duration curve for cycle ergometry. Ergonomics, 43(1), 133-141.

Moritani, T., Nagata, A., deVries, H.A. \& Muro, M. (1981). Critical power as a measure of physical work capacity and anaerobic threshold. Ergonomics, 24(5), 339-350.

Murgatroyd, S.R., Ferguson, C., Ward, S.A., Whipp, B.J. \& Rossiter, H.B. (2011). Pulmonary $\mathrm{O}_{2}$ uptake kinetics as a determinant of high-intensity exercise tolerance in humans. Journal of applied physiology (Bethesda, Md.: 1985), 110(6), 1598-1606.

Paixao, R.C., da Mota, G.R. \& Marocolo, M. (2014). Acute effect of ischemic preconditioning is detrimental to anaerobic performance in cyclists. International Journal of Sports Medicine, 35(11), 912-915.

Paton, C.D. \& Hopkins, W.G. (2001). Tests of cycling performance. Sports medicine (Auckland, N.Z.), 31(7), 489-496.

Patterson, S.D., Bezodis, N.E., Glaister, M. \& Pattison, J.R. (2015). The Effect of Ischemic Preconditioning on Repeated Sprint Cycling Performance. Medicine and science in sports and exercise, 47(8), 1652-1658.

Poole, D.C., Burnley, M., Vanhatalo, A., Rossiter, H.B. \& Jones, A.M. (2016). Critical Power: An Important Fatigue Threshold in Exercise Physiology. Medicine and science in sports and exercise, 48(11), 2320-2334.

Poole, D.C., Ward, S.A., Gardner, G.W. \& Whipp, B.J. (1988). Metabolic and respiratory profile of the upper limit for prolonged exercise in man. Ergonomics, 31(9), 1265-1279.

Rassaf, T., Totzeck, M., Hendgen-Cotta, U.B., Shiva, S., Heusch, G. \& Kelm, M. (2014). Circulating nitrite contributes to cardioprotection by remote ischemic preconditioning. Circulation research, 114(10), 1601-1610.

Saito, T., Komiyama, T., Aramoto, H., Miyata, T. \& Shigematsu, H. (2004). Ischemic preconditioning improves oxygenation of exercising muscle in vivo. The Journal of surgical research, 120(1), 111-118.

Salvador, Amadeo, F., Aguiar, R. A. De, Lisboa, F. D., Pereira, K. L., \& Caputo, F. (2016). Ischemic Preconditioning and Exercise Performance: A Systematic Review and MetaAnalysis. International Journal of Sports Physiology and Performance, 11, 4-14 
Stamler, J.S. \& Meissner, G. (2001). Physiology of nitric oxide in skeletal muscle. Physiological Reviews, 81(1), 209-237.

Tew, G.A., Ruddock, A.D. \& Saxton, J.M. (2010). Skin blood flow differentially affects near-infrared spectroscopy-derived measures of muscle oxygen saturation and blood volume at rest and during dynamic leg exercise. European Journal of Applied Physiology, 110, 1083-1089.

Vanhatalo, A., Black, M.I., DiMenna, F.J., Blackwell, J.R., Schmidt, J.F., Thompson, C., Wylie, L.J., Mohr, M., Bangsbo, J., Krustrup, P. \& Jones, A.M. (2016). The mechanistic bases of the power-time relationship: muscle metabolic responses and relationships to muscle fibre type. The Journal of physiology, 594(15), 4407-4423.

Vanhatalo, A., Doust, J.H. \& Burnley, M. (2007). Determination of critical power using a 3min all-out cycling test. Medicine and science in sports and exercise, 39(3), 548-555.

Vanhatalo, A., Fulford, J., DiMenna, F.J. \& Jones, A.M. (2010). Influence of hyperoxia on muscle metabolic responses and the power-duration relationship during severe-intensity exercise in humans: a 31P magnetic resonance spectroscopy study. Experimental physiology, 95(4), 528-540.

Whipp, B. J. (1987). Dynamics of pulmonary gas exchange. Circulation, 76(6 Pt 2), VI18-28. 


\section{FIGURE LEGENDS}

Figure 1. CP measured during the 3 min all-out test following IPC and SHAM interventions. Values are means \pm SD. * Significant difference between IPC and SHAM $(\mathrm{P}<0.05)$.

Figure 2. Predicted time to complete targets of work done $(50-250 \mathrm{~kJ})$. Values are means \pm SD. * Significant difference between IPC and SHAM $(\mathrm{P}<0.05)$.

\section{TABLE LEGENDS}

Table 1. Physiological variable responses to IPC and SHAM during the 3 min all-out test.

Table 2. Effect of the IPC and SHAM interventions on TSI responses before and during the 3 min all-out test. 


\section{Table 1}

\begin{tabular}{|c|c|c|c|}
\hline & IPC & SHAM & p value \\
\hline CP (W) & $241 \pm 65$ & $234 \pm 67$ & 0.011 \\
\hline $\mathrm{W}^{\prime}(\mathrm{kJ})$ & $17.9 \pm 3.7$ & $18.4 \pm 3.8$ & 0.355 \\
\hline TWD (kJ) & $60.8 \pm 12.7$ & $61.1 \pm 12.7$ & 0.830 \\
\hline PPO (W) & $821 \pm 186$ & $823 \pm 204$ & 0.869 \\
\hline$\dot{\mathrm{V}}_{\text {Opeak }}\left(\mathrm{L} \cdot \mathrm{min}^{-1}\right)$ & $3.93 \pm 0.67$ & $3.85 \pm 0.74$ & 0.298 \\
\hline Total $\mathrm{O}_{2}$ consumed $(\mathrm{L})$ & $10.86 \pm 1.75$ & $10.98 \pm 2.15$ & 0.719 \\
\hline$\dot{\mathrm{V}}_{2} \operatorname{MRT}(\mathrm{s})$ & $15 \pm 6$ & $15 \pm 6$ & 0.863 \\
\hline Blood [lactate] (mmol.L $\left.{ }^{-1}\right)$ & $12.7 \pm 2.1$ & $11.9 \pm 1.3$ & 0.212 \\
\hline $\mathrm{HR}_{\text {mean }}(\mathrm{bpm})$ & $163 \pm 18$ & $160 \pm 20$ & 0.257 \\
\hline $\mathrm{HR}_{\max }(\mathrm{bpm})$ & $177 \pm 9$ & $176 \pm 9$ & 0.331 \\
\hline
\end{tabular}

$\mathrm{CP}$, critical power; TWD, total work done; PPO, peak power output; MRT, mean response time; HR, heart rate. Data are mean \pm SD. 
Table 2

\begin{tabular}{lccc}
\hline & IPC & SHAM & p value \\
\hline Baseline (\%) & $72.4 \pm 3.9$ & $71.8 \pm 3.0$ & 0.480 \\
Intervention (\%) & $56.4 \pm 0.7$ & $71.7 \pm 0.9$ & 0.001 \\
Exercise - 60-s (\%) & $46.3 \pm 8.6$ & $45.1 \pm 10.5$ & 0.582 \\
Exercise - 120-s (\%) & $47.4 \pm 9.8$ & $46.0 \pm 10.9$ & 0.495 \\
End-Exercise (\%) & $47.5 \pm 10.0$ & $45.8 \pm 11.0$ & 0.371 \\
\hline
\end{tabular}

TSI, tissue saturation index. Data are mean \pm SD. 


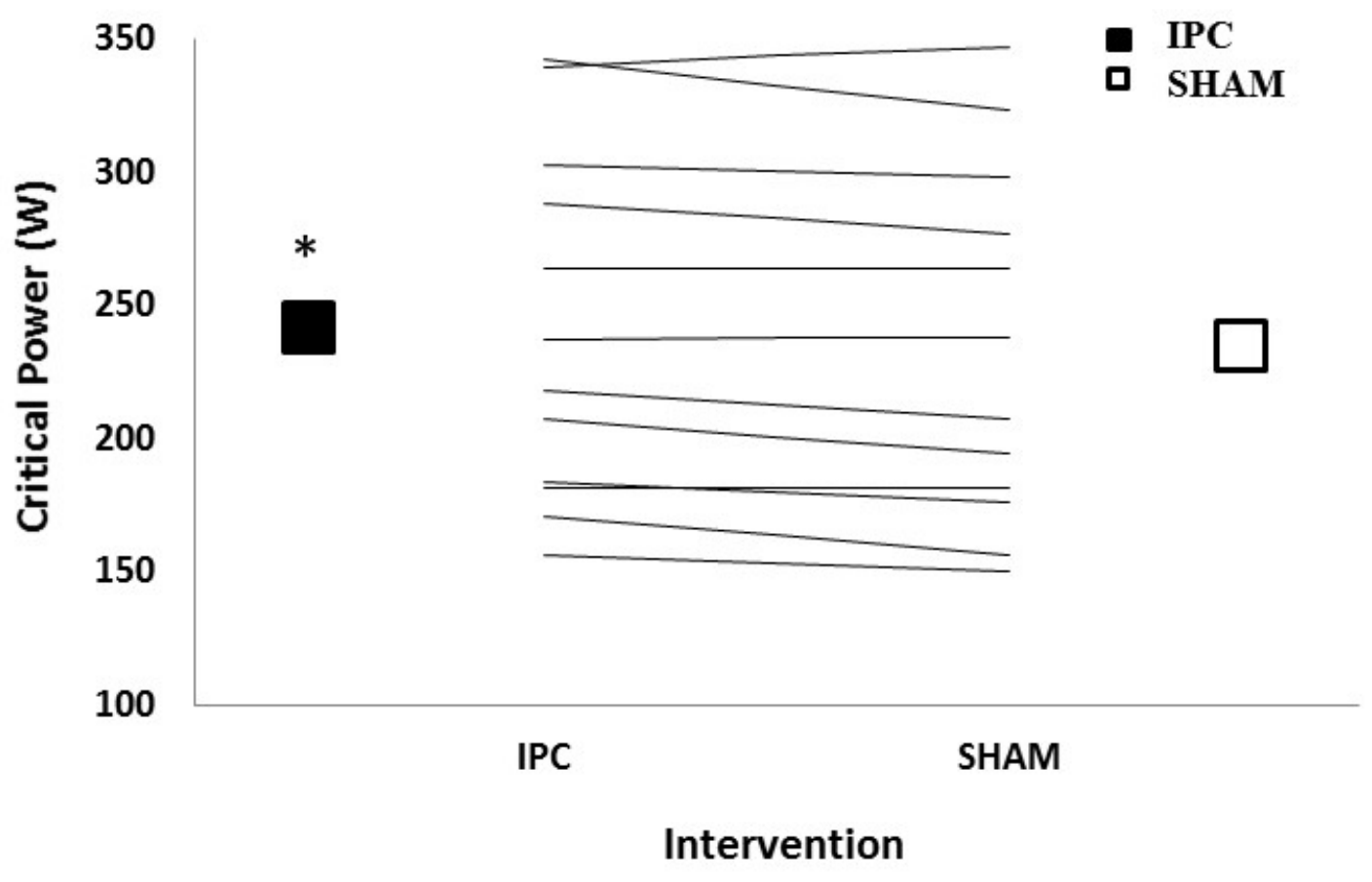

Figure 1 


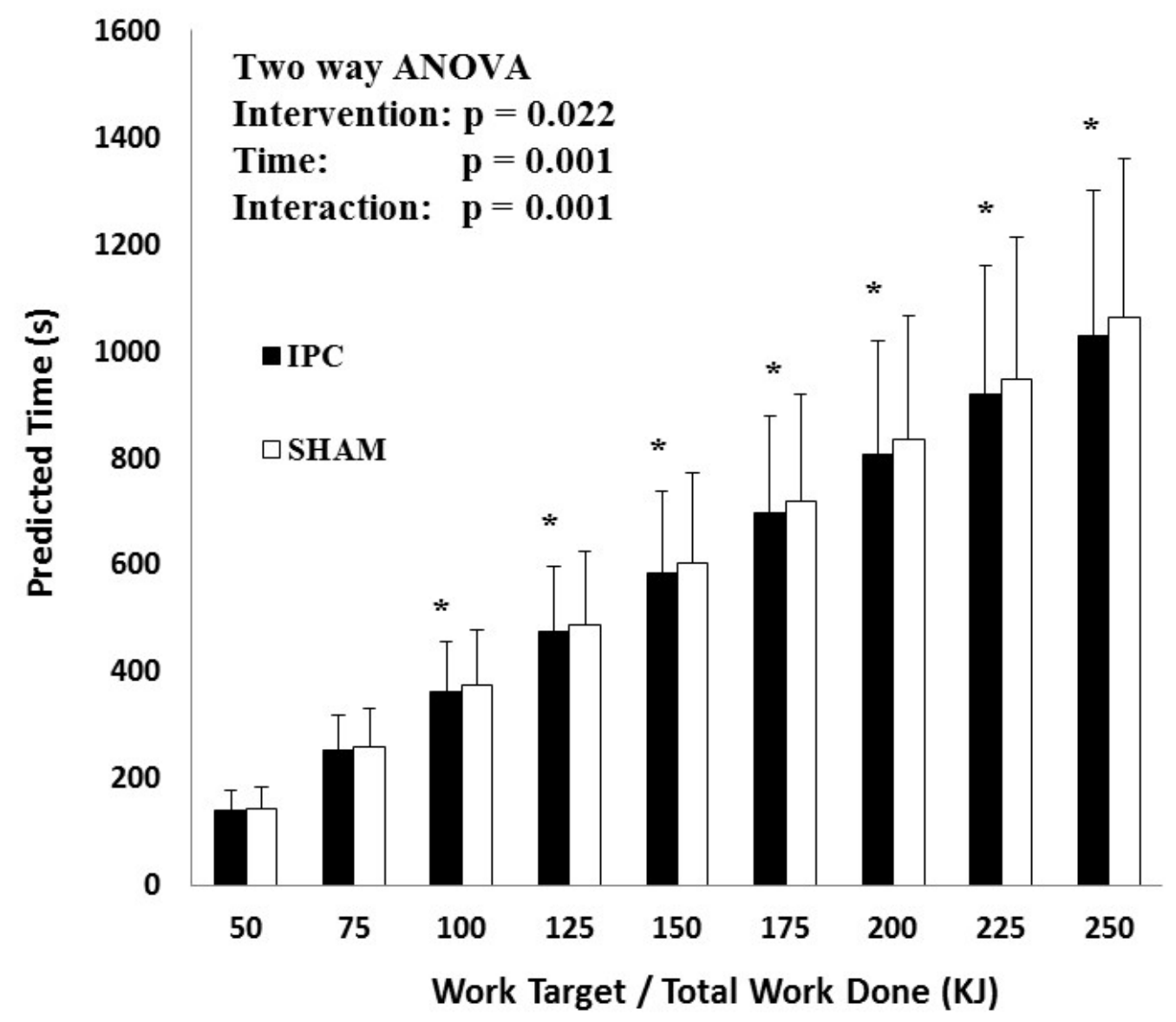

Figure 2 